\title{
Digital Topologies Revisited: An Approach Based on the Topological Point-Neighbourhood ${ }^{\star}$
}

\author{
Pavel Ptak ${ }^{1}$, Helmut Kofler ${ }^{2}$, Walter Kropatsch ${ }^{2}$
}

\author{
${ }^{1}$ Center for Machine Perception \\ CMP - Czech Technical University \\ Faculty of Electrical Engineering \\ Karlovo nam. 13 \\ 12135 Praha 2, Czech Republic \\ ptak@math.feld.cvut.cz
}

\author{
${ }^{2}$ Pattern Recognition and \\ Image Proc. Group - PRIP \\ Vienna University of Technology \\ Treitlstr. 3 , \\ A-1040 Vienna, Austria \\ $\{\mathrm{krw}, \mathrm{kof}\} @$ prip.tuwien.ac.at
}

\begin{abstract}
Adopting the point-neighbourhood definition of topology, which we think may in some cases help acquire a very good insight of digital topologies, we unify the proof technique of the results on 4-connectedness and on 8-connectedness in $\mathbb{Z}^{2}$. We also show that there is no topology compatible with 6 -connectedness. We shortly comment on potential further use of this approach.
\end{abstract}

Keywords: Image processing, digital topology, adjacency, path-connectedness and topological connectedness in $\mathbb{Z}^{2}$.

\section{Introduction and Basic Definitions}

The domain of a digital picture can be viewed as a subset of $\mathbb{Z}^{2}$, where $\mathbb{Z}$ stands for the set of integers, together with some adjacency neighbourhood structure $[2,7,6]$ assigned to each point. Thus, for instance, we may talk on 8-adjacency neighbourhood structure (in shorthand, 8-structure) if each point $(x, y) \in \mathbb{Z}^{2}$ is given the adjacency neighbourhood

$$
\begin{aligned}
& (x-1, y-1)(x, y-1)(x+1, y+1) \\
& \begin{array}{lll}
(x, y-1) & (x, y) \quad(x, y+1)
\end{array} \\
& (x-1, y+1)(x+1, y)(x+1, y+1)
\end{aligned}
$$

In the sequel, let us assume as plausible that the adjacency neighbourhood structure is homogeneous (i.e., for each $\left(x_{0}, y_{0}\right)$, the natural translation $(x, y) \rightarrow\left(x+x_{0}, y+y_{0}\right)$ is an adjacency isomorphism) and symmetric with respect to the point $(x, y)$.

* This work was supported by a grant from the Austrian National Fonds zur Förderung der wissenschaftlichen Forschung (No. S7002MAT). 
We would like to demonstrate that the point-neighbourhood definition of topology adopted here provides us with a good method for deciding when (if) there is a topology compatible with adjacency. We will present different proofs of previously known results (see $[2,6,7,9])$ and slightly extend them.

We also assume that the adjacency neighbourhood never exceeds the 8neighbourhood, but note the result holds also for any larger neighbourhood.

In the following we give some elementary definitions. In section 2 we present the different adjacency neighbourhood structures. Section 3 contains the main results. Finally some conclusions are given in section 4.

\subsection{Path-Connectedness in $S$}

Suppose that $\mathbb{Z}^{2}$ is given a (homogeneous and symmetric) neighbourhood structure. In order to refer to this structure, let us call it $\mathcal{S}$. Suppose that $p, q$ are points of $\mathbb{Z}^{2}$. By an $\mathcal{S}$-path from $p$ to $q$ we understand a finite sequence of points $p=p_{1}, p_{2}, \ldots, p_{n}=q$ such that $p_{i}$ is a neighbour of $p_{i-1}(1<i \leq n)$ in the structure $\mathcal{S}$. Let us call the points $p, q \mathcal{S}$-related if there is an $\mathcal{S}$-path from $p$ to $q$.

Let $X$ be a subset of $\mathbb{Z}^{2}$. Since the relation of being $S$-related, for a given $S$, is obviously an equivalence relation on $X$. This relation gives rise to a partition of $X$ into classes of $\mathcal{S}$-related elements. Let us call each class of this equivalence an $\mathcal{S}$-component.

A natural question arises $([8,2]$, etc.) if (when) the $\mathcal{S}$-components can be obtained as the components of a connectedness relation of a topology. Let us view basic notions we need for pursuing this question. Out of several possible definitions of (classical) topology, the definition involving the point-neighbourhood structure may best serve the purpose.

\subsection{Topological Connectedness}

Definition (Topological space): Let $P$ be a set. Let us assign to each $x \in P$ a set $\mathcal{U}(x)$ of subsets of $P$ which is subject to the following conditions:

(i) if $U \in \mathcal{U}(x)$, then $x \in U$,

(ii) if $U \in \mathcal{U}(x)$ and $U \subset V$, then $V \in \mathcal{U}(x)$,

(iii) if $U, V \in \mathcal{U}(x)$, then $U \cap V \in \mathcal{U}(x)$,

(iv) if $U \in \mathcal{U}(x)$, then there exists $V \in \mathcal{U}(x)$ such that, for each $y \in V, U \in$ $\mathcal{U}(y)$.

The set $P$ together with the assignment $\mathcal{U}(x)$, for each $x \in P$, is called a topological space. The sets $U \in \mathcal{U}(x)$ are called topological neighbourhoods of $x$. We denote the assignment $x \rightarrow \mathcal{U}(x)$ by $t$ - the topology. So we can refer to the couple $(P, t)$, meaning the corresponding topological space. 
It should be noted that this definition of topological space is equivalent to the "base-for-open-sets" definition or to the "closure" definition. This can be easily verified by a straightforward translation (see e.g. [1]). One should also observe that it is the axiom(iv) which is usually responsible for inconveniences when one looks for "suitable" topologies (this can be compensated by a possibility to apply the topological result back into the real model).

Let $(P, t)$ be a topological space defined in the sense of previous definition (i. e. via point-neighbourhoods) and let $X$ be a subset of $P$. We say that $\left(X, t_{1}\right)$ is a topological subspace of $(P, t)$ if, for each $x \in X$, the set $\mathcal{U}(x) \cap X$ is the set of all neighbourhoods of $x$ in the topology $t_{1}$. It can be seen easily that $\left(X, t_{1}\right)$ is then a topological space in its own right. Moreover, if $(P, t)$ is a topological space and $Y, V$ are subsets of $P$ with $Y \subset V$, then if $\left(Y, t_{1}\right),\left(V, t_{2}\right)$ are topological subspaces of $(P, t)$, then $\left(Y, t_{1}\right)$ is a topological subspace of $\left(V, t_{2}\right)$.

Definition (Topological connectedness): Let $(P, t)$ be a topological space. We say that $(P, t)$ is disconnected if there are two disjoint sets $R, S$ such that $R \cup S=P$ and, moreover, for each $r \in R$ and each $s \in S$ the set $R$ is a neighbourhood of $r$ and $S$ is a neighbourhood of $s$. The space $(P, t)$ is said to be connected if it is not disconnected. Finally, a subset $X$ of $(P, t)$ is said to be connected if the subspace $\left(X, t_{1}\right)$ of $(P, t)$ is connected.

Obviously, a connected topological space may have plenty of disconnected subspaces. For instance, the set $(-\infty, 0) \cup(0,+\infty)$ or the set $\{1,2,3, \ldots\}$ are obviously disconnected subspaces of the space $R$ of reals (resp. the topology induced by one of the equivalent n-norms $n=1,2, \infty)$. Also, the set $Q$ of all rational numbers is also disconnected - we can write $Q=Q_{1} \cup Q_{2}$, where $Q_{1}=$ $\{q \in Q \mid q<\sqrt{2}\}$ and $Q_{2}=\{q \in Q \mid q>\sqrt{2}\}$. The last example shows that the question on deciding about connectedness of a subspace may be sometimes nontrivial.

\section{Neighbourhood Structures on $\mathbf{Z}^{2}$}

Definition (Compatibility): Let $\mathcal{S}$ denote a given adjacency structure on $\mathbb{Z}^{2}$ and let $t$ be a topology on $\mathbb{Z}^{2}$. We say that $t$ is compatible with $\mathcal{S}$ if for each $X, X \subset \mathbb{Z}^{2}$, the set $X$ is connected (with respect to $t$ ) if and only if $X$ is $\mathcal{S}$ connected.

Let us employ the following definition (see also [3]). Let $(P, t)$ be a topological space. We say that $(P, t)$ is locally finite if each point $x \in P$ possesses the finite neighbourhood $U \in \mathcal{U}(x)$, i.e. $|U|<\infty$. Obviously, if $(P, t)$ is locally finite then each point $x \in P$ possesses a smallest neighbourhood in $t$ and this neighbourhood is finite. This follows from the closedness of the neighbourhood under the formation of intersections. An important fact in our considerations is this: If $U(x)$ is the smallest (topological) neighbourhood of $x$, then $U(x)$ must also be a neighbourhood of all points in $U(x)$. This immediately follows from the point-neighbourhood definition of topology (the axiom (iv)). 
We will now investigate all possible (homogeneous and symmetric) adjacency neighbourhood structures in $\mathbb{Z}^{2}$ (see the figure below) and formulate results on the compatible topologies. We will in turn take up the 0-adjacency, 2-adjacency, 4-adjacency, 6-adjacency and 8-adjacency.

(i)

(ii)

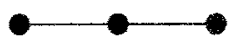

(iii)

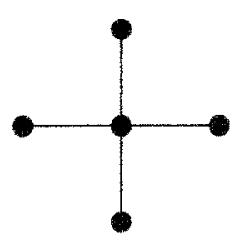

(iv)

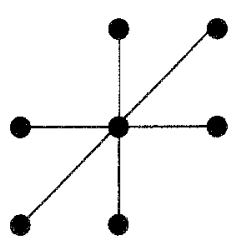

$(\mathrm{v})$

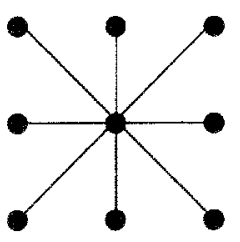

0 -adjacency (to each point $(x, y) \in \mathbb{Z}^{2}$ the only point $(x, y)$ is adjacent)

2-adjacency (to each point $(x, y) \in \mathbb{Z}^{2}$ the points $(x-1, y)$ and $(x+1, y)$ are adjacent)

4-adjacency (to each point $(x, y) \in \mathbb{Z}^{2}$ the points $(x-1, y),(x+1, y),(x, y-1),(x, y+1)$ are adjacent)

6-adjacency (to each point $(x, y) \in \mathbb{Z}^{2}$ the points $(x-1, y),(x+1, y),(x, y-1),(x, y+1)$, $(x-1, y-1),(x+1, y+1)$ are adjacent $)$

8-adjacency (to each point $(x, y) \in \mathbb{Z}^{2}$ the points $(x-1, y),(x+1, y),(x, y-1),(x, y+1)$, $(x-1, y-1),(x+1, y+1),(x-1, y+1)$, $(x+1, y-1)$ are adjacent $)$.

Remark: The $n$-adjacencies, $n$ is odd, are not homogeneous and 2-point connections (1-adjacency) can be compared only with a trivial topology like the discrete topology.

\section{Compatible Topologies on $\mathbb{Z}^{2}$}

Theorem 1: There is exactly one topology which is compatible with the o-adjacency. This topology is the discrete topology on $\mathbb{Z}^{2}$. 
Proof: The discrete topology making each point $(x, y) \in \mathbb{Z}^{2}$ a neighbourhood of $(x, y)$ is clearly compatible with 0 -adjacency.

Theorem 2: There are infinitely many topologies which are compatible with the 2-adjacency in $\mathbb{Z}^{2}$. Among those there are infinitely many, which are locally finite.

Proof: For a fixed $x \in \mathbb{Z}$, we obviously have a topology, $t_{x}$, on the subspace $T_{x}=\{(x, y) \mid y \in \mathbb{Z}\}$ which is compatible with the 2-adjacency on the set $T_{x}$. Indeed, it suffices to take $\mathcal{U}(x)=\left\{U \subset T_{x} \mid\{(x, y)\} \subset U\right\}$ provided $y$ is odd, $\mathcal{U}(x)=\left\{U \subset T_{x} \mid\{(x, y-1),(x, y),(x, y+1)\} \subset U\right\}$ provided $y$ is even. Note that the above neighbourhoods contain two types as smallest and finite neighbourhood (i.e. the 1-dimensional Marcus-Wyse topology).

Since the roles of odd and even numbers are obviously interchangeable and since the adjacency connectedness (2-connectedness) of "different levels" do not affect each other, we can take, for each $x \in \mathbb{Z}$, one of the two topologies on $T_{x}$, obtaining a topology that is compatible with 2 -adjacency. Since we have infinitely many combinations at our disposal, the result is proved.

Remark (not locally finite topologies): A question of separate purely topological curiosity may arise whether we can construct, for the 2-adjacency, a topology which is compatible with the adjacency and which is not locally finite. This seems to be possible - the standard ultrafilter construction can be applied in this case or more easily the Frechet-filter of the infinite intervals $\{x \in \mathbb{Z} \mid x \geq z\}$, $z \in \mathbb{Z}$. These topologies are, however, hardly relevant to digital pictures.

Let us now consider the 4-adjacency. The following result, which is due to [9] and [6] is in force. We will show how one obtains this result in the pointneighbourhood formalism. The little auxiliary results stated as Observations 3.1, 3.2 may be of certain value in their own right.

Theorem 3: There are 2 topologies which are compatible with the 4-adjacency - the 2-dimensional Marcus-Wyse topologies $\tau$ [9]:

$$
U \in \tau \equiv\left\{\begin{array}{lll}
U(x, y) & : & \text { if } x+y \text { is even } \quad \text { (resp.odd), } \\
\{(x, y)\} & : \text { else }
\end{array}\right.
$$

with $U(x, y)=\{(x, y),(x, y-1),(x, y+1),(x+1, y),(x-1, y)\}$.

Proof: The result easily follows from the following two observations.

Observation 3.1: Each topology which is compatible with 4-adjacency is locally finite. Moreover, if $U(x, y)$ is the smallest neighbourhood of $(x, y)$ in a topology compatible with 4 -adjacency, then

$$
U(x, y) \subseteq\{(x, y),(x, y-1),(x, y+1),(x+1, y),(x-1, y)\} .
$$

Proof: Let $(x, y) \in \mathbb{Z}^{2}$ and let us show that the point $(x, y)$ must have a finite neighbourhood. 
Let $X_{1}=\{(x, y)\}$ and $X_{2}=\left\{(u, v) \in \mathbb{Z}^{2}|| x-u|+| y-v \mid \geq 2\right\}$, see Fig. 1.

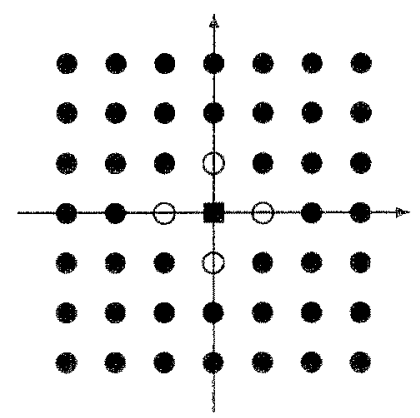

Fig. 1. The two sets $X_{1}=\{\boldsymbol{\square}\}$ and $X_{2}=\{\bullet\}$

Then both $X_{1}$ and $X_{2}$ are 4-connected but $X_{1} \cup X_{2}$ is not. It follows that there is a topological neighbourhood of $(x, y)$ in any topology compatible with 4-adjacency which is disjoint with $X_{2}$. In other words, there is a topological neighbourhood, $U(x, y)$, such that $U(x, y) \subseteq \mathbb{Z}^{2}-X_{2}$. This is what we wanted to show.

Observation 3.2: Let $(x, y) \in \mathbb{Z}^{2}$ and let $t$ be a topology compatible with 4 -adjacency. Let $U(x, y)$ be the smallest neighbourhood of $(x, y)$ in $t$. Then either $U(x, y)=\{(x, y)\}$ or $U(x, y)=\{(x, y),(x, y-1),(x, y+1),(x+1, y)$, $(x-1, y)\}$.

Proof: By the previous observation, $U(x, y) \subseteq\{(x, y),(x, y-1),(x, y+1)$, $(x+1, y),(x-1, y)\}$. Suppose that $U(x, y) \neq\{(x, y)\}$. Then, without a loss of generality, $(x-1, y) \in U(x, y)$. Suppose now that $(x, y+1) \notin U(x, y)$ (again, one argues analogously if there is another edge than $(x, y+1)$ outside of $U(x, y))$. Since the set $\{(x-1, y),(x, y+1)\}$ is not 4 -connected and the set $\{(x, y),(x, y+1)\}$ is 4-connected, we infer that the smallest neighbourhood of $(x, y+1)$, some set $U(x, y+1)$, must not contain the point $(x-1, y)$ and must contain the point $(x, y)$. Consequently considering other edges analogously,

$$
U(x, y) \cap U(x, y+1)=\{(x, y)\} .
$$

But since $U(x, y+1)$ is a topological neighbourhood, it must be also a topological neighbourhood of the point $(x, y)$. It follows that $U(x, y) \cap U(x, y+1)$ must be a topological neighbourhood of the point $(x, y)$.

But $U(x, y) \cap U(x, y+1)=\{(x, y)\}$ which is a contradiction. This completes the proof of Observation 3.2 .

Let us return to the proof of Theorem 3 . Let $t$ be a topology which is compatible with the 4-adjacency. It is obvious that the singleton sets $\{(x, y)\}$ cannot 
constitute the neighbourhoods for all $(x, y) \in \mathbb{Z}^{2}$ (we would obtain the discrete topology; the discrete topology is obviously not compatible with 4-adjacency). It is also obvious that the sets

$$
\{(x, y),(x-1, y),(x+1, y),(x, y-1),(x, y+1)\}
$$

cannot be the smallest neighbourhoods for all $(x, y) \in \mathbb{Z}^{2}$ (we would not have a topology at all). Thus, in every topology compatible with 4-adjacency we must have, for some points, both the singleton smallest neighbourhoods and the "starlike" neighbourhoods. Having found the necessary conditions for a compatible topology, the rest consists of an easy inductive argument already presented in [9].

Choose, for instance, the point $(0,0)$. Then either the set $U_{1}(0,0)=\{(0,0)$, $(-1,0),(1,0),(0,1),(0,-1)\}$ or the set $U_{2}(0,0)=\{(0,0)\}$ must be the smallest neighbourhood of $(0,0)$.

In the former case, the smallest neighbourhoods of the points $(-1,0),(1,0)$, $(0,-1),(0,1)$ must necessarily be the singleton sets, the smallest neighbourhoods of the points $(-1,-1),(-1,1),(1,-1),(1,1)$ must be the "star" sets, and so on. In the latter case, the smallest neighbourhoods of the points $(-1,0),(1,0)$, $(0,-1),(0,1)$ must be the "star" sets, the smallest neighbourhoods of the points $(-1,-1),(-1,1),(1,-1),(1,1)$ must be the singleton sets, and so on.

Consequently, there are only two topologies on $\mathbb{Z}^{2}$ which are compatible with the 4-adjacency - either the Marcus-Wyse topology or the topology obtained from it by the shift $(x, y) \rightarrow(x, y+1)$. The Marcus-Wyse topology allows for a simple description as also the present consideration shows: the smallest neighbourhood of $(x, y)$ is a singleton set provided $x+y$ is even, and the smallest neighbourhood of $(x, y)$ is a star set provided $x+y$ is odd.

The rest would consist in checking that, indeed, the Marcus-Wyse topology is compatible with 4-adjacency. This is not difficult and has been done in detail in [9]. The proof is complete.

Let us now consider the 6-adjacency (see the schema in the figure (iv)). In this case the search for compatible topology would be in vain.

Theorem 4: There is no topology on $\mathbb{Z}^{2}$ which is compatible with the 6-adjacency.

Proof: Suppose that $t$ is a topology compatible with the 6-adjacency. By the very same reasoning we employed in Observations $3.1,3.2$ we can derive the following results:

(i) The topology $t$ is locally finite,

(ii) If $(x, y) \in \mathbb{Z}^{2}$, then the smallest neighbourhood of $(x, y)$ in $t$ is either the singleton set $\{(x, y)\}$ or the whole 6 -star set

$$
\{(x, y),(x-1, y),(x+1, y),(x, y-1),(x, y+1),(x-1, y-1),(x+1, y+1)\} .
$$


There must be a point in $\mathbb{Z}^{2}$ with the proper 6-adjacency neighbourhood. Let us denote it again by $(x, y)$. It follows that the points $(x+1, y)$ and $(x+1, y+1)$ must have the singleton set neighbourhoods. This is absurd since the set $\{(x+1, y),(x+1, y+1)\}$ is 6 -connected. The proof is complete.

The following corollary to the previous result can be viewed as another proof of the result by Chassery [2] and L. Latecki [7].

Theorem 5: There is no topology on $\mathbb{Z}^{2}$ which is compatible with the 8-adjacency.

Proof: It is easily seen that if $\mathcal{S}_{1}$ and $\mathcal{S}_{2}$ are two adjacency relations on $\mathbb{Z}^{2}$ and $\mathcal{S}_{2}$ is finer then $\mathcal{S}_{1}$, then the absence of a locally finite compatible topology for $\mathcal{S}_{1}$ implies the absence of a locally finite compatible topology for $\mathcal{S}_{2}$. Since there is no topology compatible with 6-adjacency, there is no topology compatible with the 8-adjacency.

\section{Conclusion}

We have completed the tour over all possible "nice" adjacencies in $\mathbb{Z}^{2}$. Presumably the next step is testing the suitability of the point-neighbourhood approach is the examination of concrete (finite) configurations of points in $\mathbb{Z}^{2}$ and, of course, the digital topologies in $\mathbb{Z}^{n}$ for $n \geq 3$. We intend to pursue this elsewhere. It should be observed, however, that it does not seem possible to analyze $\mathbb{Z}^{n}$ with the help of viewing $\mathbb{Z}^{n}$ as a Cartesian product of $\mathbb{Z}^{m}$ for $m$ smaller than $n$. This can be graphically seen even for $\mathbb{Z}^{2}$. Indeed, none of the adjacencies on $\mathbb{Z}^{2}$ with the exception of the discrete one is obtained as a "Cartesian product" of adjacencies on $\mathbb{Z}$. We may however obtain nontrivial (non-homogeneous) adjacencies this way or, in other words, homogeneity may be a too restricting property for digital topologies. In particular if the data stem from a projection of a higher dimensional space (e.g. 3-dim) onto a lower dimension (e.g. 2-dim). In the case of digital images, we may seek the topological properties of the 3dim objects in the 2-dim image rather than establishing adjacency across object boundaries. Such occluding boundaries represent discontinuities of the image function and adjacent pixels may correspond to 3-dim points of different objects which are far apart in reality.

If, for instance, we take the 2-adjacency on $\mathbb{Z}$ and multiply it with each other, we obtain an adjacency on $\mathbb{Z}^{2}$ which is the 4-adjacency on the points of the type (even, even), the 2-adjacency on the points of the type (even,odd) and (odd, even) - in the former case vertically and in the latter case horizontally, and the 1-adjacency on the points of the type (odd,odd). Since the 2-adjacency on $\mathbb{Z}$ allows for a compatible topology, so does our "mixed" adjacency on $\mathbb{Z}^{2}$, which directly leads to the abstract cellular complexes of Kovalevsky [3, 4].

This survey is part of an ongoing research project with two primary goals to extend the results to higher dimensions and to irregular adjacency neighbourhood structures [5]. 


\section{References}

1. E. Cech: Topological Spaces, Interscience, Wiley, New York, 1966.

2. J.M. Chassery: Connectivity and consecutivity in digital pictures, Computer Graphics and Image Processing 9, 294-300, 1979.

3. E. Khalimsky, R. Kopperman and P.R. Meyer: Computer graphics and connected topologies on finite ordered sets, Topology Appl. 36, 117, 1980.

4. V.A.Kovalevsky: Finite topology as applied to image analysis, Computer Vision, Graphics and Image Processing 46, 141-161, 1989.

5. W. G. Kropatsch: Equivalent Contraction Kernels and the Domain of Dual Irregular Pyramids, Technical Report PRIP-TR-042, TU-Vienna, 1996.

6. L.Latecki: Digitale und Allgemeine Topologie in der bildhaften Wis* sensrepräsentation, Ph.D.-Thesis, Hamburg, 1992.

7. L. Latecki: Topological connectedness and 8-connectedness in digital pictures, Computer Vision, Graphics and Image Processing: Image Understanding 57, 261-262, 1993.

8. A. Rosenfeld: Digital topology, Am. Math. Monthly 86, 621-630, 1979.

9. F. Wyse and D. Marcus et al.: Solution to Problem 5712, Am. Math. Monthly 77, $1119,1970$. 